\title{
Comparative Proteomic Analysis of Anti- Cancer Mechanism by Periplocin Treatment in Lung Cancer Cells
}

\author{
Zejun Lu Qu $^{\mathrm{a} d}$ Q Song ${ }^{\mathrm{b}, \mathrm{d}}$ Jinliang Yang ${ }^{\mathrm{c}}$ Xiangfei Zhao Xinhong Zhang $^{\mathrm{a}}$ Ping Yang ${ }^{\mathrm{a}}$ \\ Jingbo Kang ${ }^{\mathrm{a}}$
}

aDepartment of Radiation Oncology, Naval General Hospital of PLA; ${ }^{b}$ Department of Gynaecology and Obstetrics, the General Hospital of Chinese People's Armed Police Force, Beijing; 'State Key Laboratory of Biotherapy and Cancer Center, West China Hospital, Sichuan University, Chengdu, China; ${ }^{d}$ These authors contributed equally to this work

\section{Key Words}

Lung cancer • Periplocin • Two-demensional electrophoresis • Proteome

\begin{abstract}
Background: Periplocin is used for treatment of rheumatoid arthritis, reinforcement of bones and tendons, palpitations or shortness of breath and lower extremity edema in traditional medicine. Our previous findings suggested that periplocin could inhibit the growth of lung cancer both in vitro and in vivo. But the biological processes and molecular pathways by which periplocin induces these beneficial effects remain largely undefined. Methods: To explore the molecular mechanisms of periplocin involved in anti-cancer activity, in the present study the protein profile changes of human lung cancer cell lines A549 in response to periplocin treatment were investigated using the proteomics approaches (2-DE combined with MS/MS). Western blot was employed to verify the changed proteins. Interactions between changed proteins were analyzed by STRING. Results: 29 down-regulated protein species named GTPbinding nuclear protein Ran (RAN), Rho GDP-dissociation inhibitor 1 (ARHGDIA), eukaryotic translation initiation factor 5A-1 (EIF5A) and Profilin-1(PFN1), and 10 up-regulated protein species named Heat shock cognate $71 \mathrm{kDa}$ protein (HSPA8), $10 \mathrm{kDa}$ heat shock protein (HSPE1), and Cofilin-1(CFL-1) were identified. Among them, GTP-binding nuclear protein Ran (RAN) and Rho GDP-dissociation inhibitor 1 (ARHGDIA) were the most significantly changed (over tenfold). The proteasome subunit beta type-6 (PSMB6), ATP synthase ecto- $\alpha$-subunit (ATP5A1), Aldehyde dehydrogenase 1 (ALDH1) and EIF5A were verified by immunoblot assays to be dramatically down-regulated. By STRING bioinformatics analysis revealing interactions and signaling networks it became apparent that the proteins changed they are primarily involved in transcription and proteolysis. Conclusion: Periplocin inhibited growth of lung cancer by down-regulating proteins, such as ATP5A1, EIF5A, ALDH1 and PSMB6. These findings may improve our understanding of the molecular mechanisms underlying the anti-cancer effects of periplocin on lung cancer cells.
\end{abstract}




\section{Introduction}

Lung cancer remains the leading cause of cancer death in both men and women even though an extensive list of risk factors has been well-characterized. Chemotherapy, including camptothecin, taxane, platin, and vinca alkaloid derivatives, has only a limited effect [1]. The large number of cases and poor survival rates under current therapies necessitate the search for novel drugs for lung cancer. Recent researches have demonstrated that natural products exhibited anti-cancer effects [2]. As many as $40 \%$ of anti-cancer drugs in use are derived from natural products or its derivatives, such as vinca (or Catharanthus) alkaloids, epipodophyllotoxins, taxanes, and camptothecins. Cardiac glycosides, known as ligands of the sodium pump, have been widely used for the treatment of heart failure, emetics, diuretics and abortifacients [3]. Recently, more and more researchers have demonstrated that cardiac glycosides, including digoxin, digitoxin, and oleandrin, showed anti-cancer activity in vitro and in vivo, which have indicated the possibility of developing this class of compound as chemotherapeutic agents in oncology [4]. For instance, the death rate and cancer recurrence turned out to be lower in women with breast cancer treated with digitalis than in nontreated patients [5]. In addition, Anvirzel, derived from the plant Nerium Oleander, has been utilized to treat advanced malignancies in phase I clinical trial [6]. Periplocin is a cardiac glycoside derived from cortex periplocae which is used for treatment of rheumatoid arthritis and reinforcement of bones and tendons in traditional Chinese medicine. Our previous study suggested that periplocin could inhibit the growth of lung cancer both in vitro and in vivo, which could be attributed to the inhibition of proliferation and the induction of apoptosis signaling pathway, such as AKT and ERK [7]. However, because of the complex anti-cancer mechanisms underlying the natural products, the molecular targets through which periplocin acts have not yet been fully determined.

In the present study, 2DE-MS strategy was utilized to analyze the alterations in protein profile of human lung cancer cell lines A549 following periplocin treatment with the aim to improve the understanding of the underlying anti-cancer molecular mechanisms of periplocin on lung cancer.

\section{Materials and Methods}

\section{Cell culture and treatment}

The human lung cancer cell line A549 was obtained from the American Type Culture Collection (ATCC, Manassas, VA). Cells were cultured in DMEM (Life Technologies, Bedford, MA) containing 10\% heatinactivated fetal bovine serum, 100 units $/ \mathrm{ml}$ penicillin and $100 \mathrm{units} / \mathrm{ml}$ streptomycin in a humid chamber at $37^{\circ} \mathrm{C}$ under $5 \% \mathrm{CO}_{2}$ in atmosphere. Periplocin was provided by Research Institute of Plant Application and Development (Chengdu, China). The periplocin stock was diluted in DMEM (containing 10\% FBS) when required for assays. For 2-DE analysis, A549 cells were treated with $5.74 \times 10^{-7} \mathrm{~mol} / \mathrm{l}$ periplocin for $24 \mathrm{~h}$. Control cells were cultured in a medium containing equal amount of NS instead of periplocin solution. The cells were collected by centrifugation and washed twice with PBS, and were subsequently transferred to sterile plastic tubes for storage at $-80^{\circ} \mathrm{C}$ until use.

Two-demensional electrophoresis (2-DE) and image analysis

2-DE was performed as described previously [8]. Briefly, cells were lysed in lysis buffer (8M urea, 2M thiourea, 4\% CHAPS, 100mM DTT, 0.2\% pH 3-10 ampholyte, BioRad, Hercules, CA) containing 1\% protease inhibitor cocktail 8340 (104 mM AEBSF, $80 \mu \mathrm{M}$ Aprotinin, $4 \mathrm{mM}$ Bestatin, $1.4 \mathrm{mM}$ E-64, 2 mM Leupeptin, 1.5mM Pepstatin A) (Sigma, St Louis, MO, USA). Samples were kept on ice and sonicated in six cycles of 15 $\mathrm{s}$, each consisting of $5 \mathrm{~s}$ sonication followed by a $10 \mathrm{~s}$ break, and then held for $30 \mathrm{~min}$ on ice with occasional vortex mixing. After centrifugation at $14,000 \mathrm{rpm}$ for $1 \mathrm{~h}$ at $4^{\circ} \mathrm{C}$, supernatant was collected and protein 
concentrations were determined using the DC protein assay kit (Bio-Rad). Protein samples (1mg) were applied to IPG (immobilized pH gradient) strip (17 cm, pH 3-10 NL, Bio-Rad) using a passive rehydration method. The second dimension was performed using $12 \%$ SDS-PAGE at $30 \mathrm{~mA}$ constant current per gel after IEF and equilibration. The gels were stained using CBB R-250 (Merck, Germany) and scanned with a BioRad GS-800 scanner. 2-DE analyses were independently repeated three times. The maps were analyzed by PDQuest software Version 6.1 (Bio-Rad). The quantity of each spot in a gel was normalized as a percentage of the total quantity of all spots in that gel and evaluated in terms of O.D. For statistical analysis, paired t-test was performed to compare data from the three repeated experiments. Only such spots that showed consistent and significant differences (over 1.5 -fold, $\mathrm{p}<0.05$ ) were selected for analysis with MS.

\section{In-Gel Digestion}

In-gel digestion of proteins was carried out using mass spectrometry grade Trypsin Gold (Promega, Madison, WI) according to the instructions given. $10 \mathrm{ng} / \mu \mathrm{l}$ trypsin was dissolved $20 \mathrm{mmol} / \mathrm{L} \mathrm{NH}_{4} \mathrm{HCO}_{3}$. Spots were cut out of the gel (1-2 mm diameter) using a razor blade, and destained twice with $100 \mathrm{mM}$ $\mathrm{NH}_{4} \mathrm{HCO}_{3} / 50 \%$ acetonitrile $(\mathrm{ACN})$ at $37^{\circ} \mathrm{C}$ for $45 \mathrm{~min}$ in each treatment. After drying, the gels were preincubated in 10-20 $\mu \mathrm{L}$ trypsin solution for $1 \mathrm{~h}$. Then $15 \mu \mathrm{L}$ digestion buffer was added $(40 \mathrm{mM}$ $\mathrm{NH}_{4} \mathrm{HCO}_{3} / 10 \% \mathrm{ACN}$ ) to cover each gel and incubated overnight at $37^{\circ} \mathrm{C}$. Tryptic digests were extracted using MilliQ water initially, followed by two times extraction with $50 \%$ ACN/5\% trifluoroacetic acid (TFA) for $1 \mathrm{~h}$ each time. The combined extracts were dried in a vacuum concentrator at room temperature. Samples were then subjected to mass spectrometric analysis.

\section{MS/MS analysis and protein identification}

Mass spectra were acquired using a Q-TOF mass spectrometer (Micromass, Manchester, UK) fitted with an ESI or MALDI source (Micromass). MS/MS analysis was performed as described previously [9]. The MS/MS data were acquired and processed using MassLynx V 4.1 software (Micromass), and were converted to PKL files by the ProteinLynx 2.2.5 software (Waters). The PKL files were analyzed using the MASCOT search engine (http://www.matrixscience.com). Search parameters were defined as follows: Database, Swiss-Prot; taxonomy, Homo sapiens; enzyme, trypsin; and allowance of one missed cleavage. Carbamidomethylation was selected as a fixed modification and oxidation of methionine was allowed to be variable. The peptide and fragment mass tolerance were set at 0.1 and $0.05 \mathrm{Da}$, respectively. Only proteins with at least one peptide exceeding their score threshold ( $p<0.05)$, and with their MW and pI consistent with the gel regions from which the spots were excised, were considered to be positively identified. For proteins identified by a single peptide and with a score higher than 40 (lower were discarded), their spectra were inspected.

\section{Immunoblot}

Cells were cracked by the RIPA lysis buffer ( $50 \mathrm{mM}$ Tris-HCl (pH 7.4), 1\%NP-40, $0.25 \%$ Na-deoxycholate, $150 \mathrm{mM} \mathrm{NaCl}, 1 \mathrm{mM}$ EDTA, $1 \mathrm{mM}$ PMSF, $1 \mathrm{mg} / \mathrm{ml}$ Aprotinin, $1 \mathrm{mM} \mathrm{Na}_{3} \mathrm{VO}_{4}, 1 \mathrm{mM} \mathrm{NaF}$ ). Then proteins were suspended in Lammli sample buffer and centrifuged at $15,000 \mathrm{rpm}$ for $30 \mathrm{~min}$. The supernatant was collected. Ten $\mu \mathrm{g}$ of each protein sample was loaded per well and separated with $12.5 \%$ SDS-PAGE. The proteins in gel were electroblotted onto PVDF membranes (Millipore) by wet blotting. After incubation in blocking buffer ( $1 \times$ TBS, $0.1 \%$ Tween-20, and $5 \% \mathrm{w} / \mathrm{v}$ dry nonfat milk) for $1 \mathrm{~h}$ at room temperature, membranes were incubated with primary antibodies (mouse-anti-human PSMB6, mouse-anti-human ALDH1, rabbit-anti-human $\beta$-actin (Santa Cruz Biotechnology, USA), rabbit-anti-human ATP5A1 (Sigma, St Louis, MO, USA) and rabbit-anti-human EIF5A (Epitomics Biotechnology, USA)). Then the membrane was incubated with secondary antibodies for $45 \mathrm{~min}$ at room temperature. Reactive bands were detected by enhanced chemiluminescence (Amersham Biosciences Corp, Piscataway, NJ).

Analysis of the signaling network and protein interactions

Analysis of the signaling network and protein-protein interactions was performed with the search tool STRING version 8.2. STRING (http://string-db.org/) is a database of known and predicted protein interactions. The interactions include direct (physical) and indirect (functional) associations [10]. 
Fig. 1. A representative 2 DE gel of periplocin- and NS-treated A549 cells. Gels were stained with Coomassie brilliant blue R250. Differential expression protein spots were labeled with numbers.

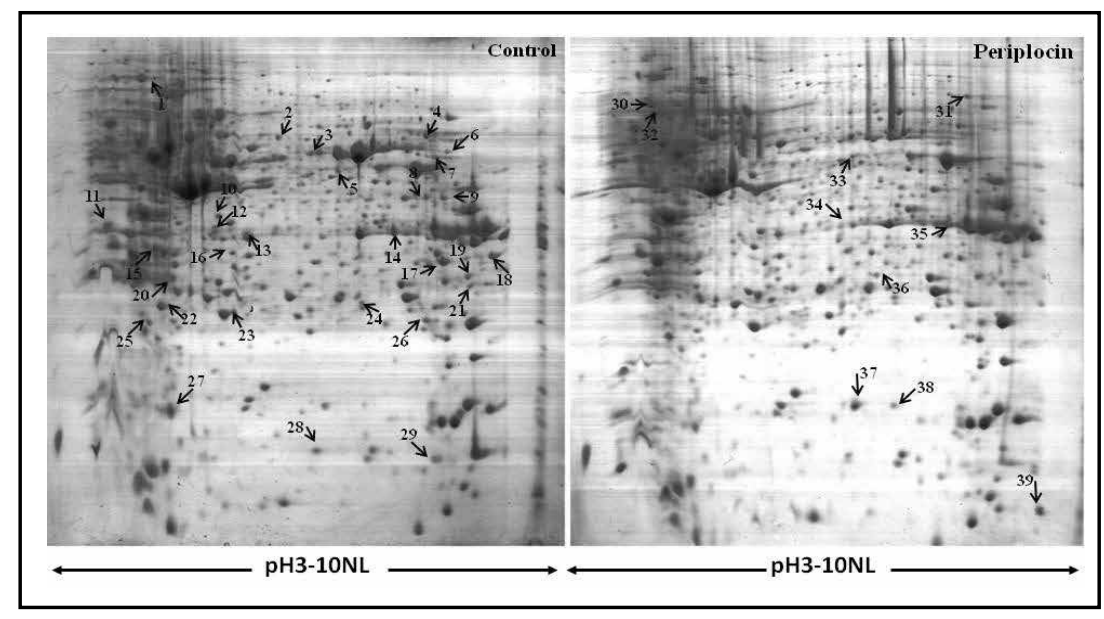

Fig. 2. Enlargement of 6 selected regions as examples showing spots which dysregulate in this study. Protein spot discrepancies were arrowed and marked with number.

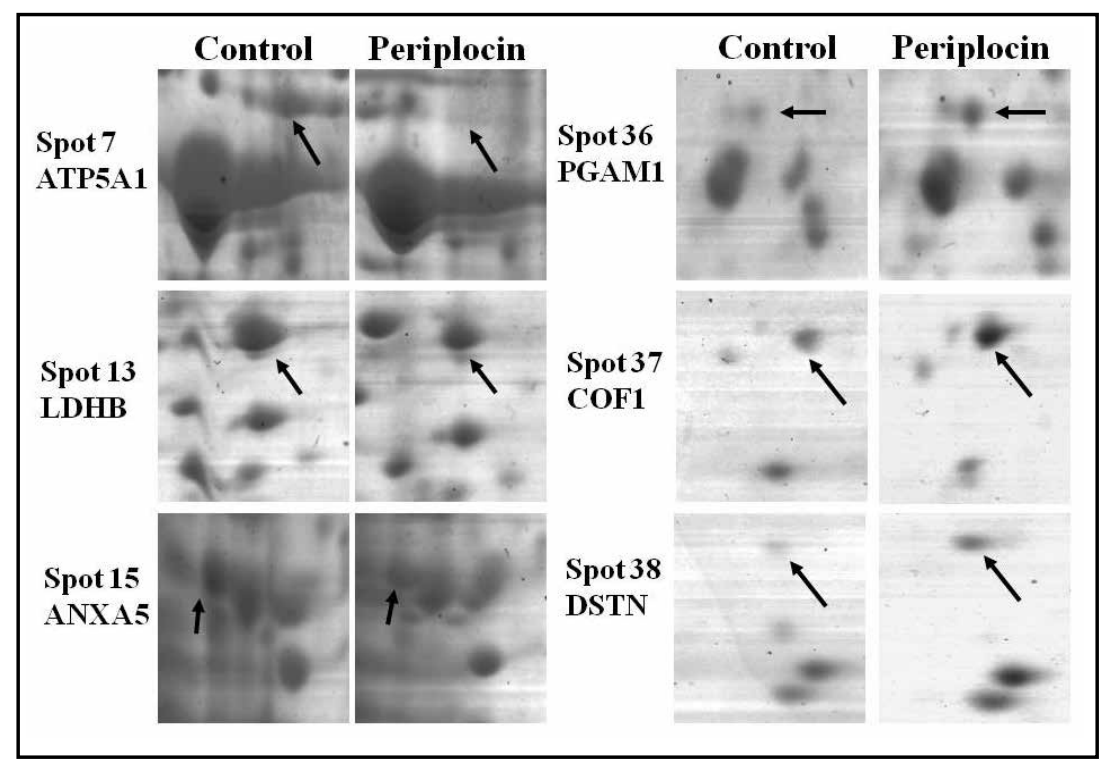

\section{Results}

\section{2-DE profiling of the differentially expressed proteins}

Protein expression in A549 cells following treatment with periplocin $\left(5.74 \times 10^{-7} \mathrm{~mol} / \mathrm{l}\right)$ and in control cells was examined by 2-DE. A pair of representative 2-DE maps is shown in Fig. 2. Proteins extracted from A549 cells treated with either periplocin or NS were resolved by 2 -DE and visualized by CBB R-250 staining. Monitor protein spots with p-value of $<0.05$ by Student's t-test and reproducible changes in intensity by more than 1.5 -fold were subjected to the analysis by MS/MS spectrometry. A total of 53 protein spots were found to be differentially expressed between periplocin and NS treated cells; of those, 39 proteins were successfully identified by MS/MS (Table 1). 29 proteins were downregulated and 10 proteins were upregulated by periplocin (Figs. 1 and 2).

\section{Protein identification and functional classification}

The 39 identified proteins (Fig. 1) are listed in Table 1. The MS/MS data, including the mass values, the intensity, and the charge of the precursor ions, were analyzed against SWISSPROT protein database with a licensed copy of the MASCOT 2.0 program. A representative MS/MS map of spot \#7 is shown in Fig. 3. The charged ion of $812.9689 \mathrm{~m} / \mathrm{z}$ and its fragment ions revealed the peptide sequence R.TGAIVDVPVGEELLGR.F, which is a part of the sequence of protein ATP5A1. Gene Ontology (G0) (www.geneontology.org/) is a useful bioinformatics 
Table 1. Identification of proteins differentially expressed between periplocin and NS treated A549 cells. The letters a-f mean: $a$ and $b$ proteins gene name and ID from ExPASy database; $c$ Theoretical molecular weight $(\mathrm{kDa})$ and $\mathrm{pI}$ from the ExPASy database. $d$ Probability-based Mowse scores. e Number of unique peptides identified by MS/MS sequencing and sequence coverage. f Average expression level in periplocin treated A549 cells compared with control cells. g N/A, not applicable because the spots on one of the paired gels were too weak or nondetectable

\begin{tabular}{|c|c|c|c|c|c|c|c|c|}
\hline $\begin{array}{l}\text { Spot } \\
\text { no. }\end{array}$ & Protein Description & $\begin{array}{c}\text { Gene } \\
\text { name }^{a}\end{array}$ & Function & $\begin{array}{c}\text { Acce.no. } \\
\text { b }\end{array}$ & $\begin{array}{c}\text { Theoreticals } \\
\text { Mr/PI }\end{array}$ & $\begin{array}{c}\text { Score } \\
\text { d }\end{array}$ & $\begin{array}{c}\text { Cove. } \\
\text { e }\end{array}$ & $\begin{array}{c}\text { Fold } \\
\text { changef }^{\mathrm{f}}\end{array}$ \\
\hline 1 & Ubiquitin carboxyl-terminal hydrolase 5 & USP5 & $\begin{array}{l}\text { Transcription } \\
\text { regulation }\end{array}$ & P45974 & $96638 / 4.91$ & 135 & $22 \%$ & $\downarrow 3.2 \pm 1.1$ \\
\hline 2 & T-complex protein 1 subunit alpha & TCP1 & Molecular chaperone & P17987 & $60819 / 5.8$ & 858 & $53 \%$ & $\downarrow 4.1 \pm 1.4$ \\
\hline 3 & Retinal dehydrogenase 1 & ALDH1 & Metabolism & P00352 & $55454 / 6.3$ & 199 & $29 \%$ & $\downarrow 7.1 \pm 3.8$ \\
\hline 4 & UDP-glucose 6-dehydrogenase & $\mathrm{UGDH}$ & Metabolism & 060701 & $55674 / 6.73$ & 521 & $48 \%$ & $\downarrow 5.4 \pm 2.6$ \\
\hline 5 & S-adenosylmethionine synthase isoform type-2 & MAT2A & Metabolism & P31153 & $43975 / 6.02$ & 142 & $16 \%$ & $\downarrow 8.6 \pm 3.7$ \\
\hline 6 & Heat shock cognate $71 \mathrm{kDa}$ protein & HSPA8 & Molecular chaperone & P11142 & $71082 / 5.37$ & 92 & $8 \%$ & $\downarrow 2.2 \pm 0.9$ \\
\hline 7 & ATP synthase subunit alpha & ATP5A1 & Metabolism & P25705 & $59828 / 9.16$ & 187 & $22 \%$ & $\downarrow 2.1 \pm 0.6$ \\
\hline 8 & Mitochondrial import receptor subunit TOM40 homolog & T0MM40 & Protein transport & 096008 & $38211 / 6.79$ & 780 & $47 \%$ & $\downarrow 3.4 \pm 1.4$ \\
\hline 9 & Phosphoserine aminotransferase & PSAT1 & Metabolism & Q9Y617 & $40796 / 7.56$ & 249 & $44 \%$ & $\downarrow 4.5 \pm 2.2$ \\
\hline 10 & Eukaryotic translation initiation factor 3 subunit I & EIF3I & Translation regulation & Q13347 & $36878 / 5.38$ & 130 & $46 \%$ & $\downarrow 9.5 \pm 3.7$ \\
\hline 11 & Nascent polypeptide-associated complex subunit alpha & NACA & Protein transport & Q13765 & $23370 / 4.2$ & 298 & $32 \%$ & $\downarrow \mathrm{N} / \mathrm{Ag}^{\mathrm{g}}$ \\
\hline 12 & $\begin{array}{l}\text { Guanine nucleotide-binding protein } \mathrm{G}(\mathrm{I}) / \mathrm{G}(\mathrm{S}) / \mathrm{G}(\mathrm{T}) \text { subunit } \\
\text { beta-2 }\end{array}$ & GNB2 & Metabolism & P62879 & $38048 / 5.6$ & 96 & $28 \%$ & $\downarrow 9.7 \pm 4.1$ \\
\hline 13 & L-lactate dehydrogenase B chain & LDHB & Metabolism & P07195 & $36900 / 5.71$ & 243 & $32 \%$ & $\downarrow 6.2 \pm 2.9$ \\
\hline 14 & Aldose reductase & AKR1B1 & Metabolism & P15121 & $36230 / 6.51$ & 209 & $59 \%$ & $\downarrow 9.2 \pm 3.2$ \\
\hline
\end{tabular}

tool to facilitate interpretation of proteomics data. GO analysis showed that periplocin could affect proteins which regulate a broad range of cellular processes and functions, such as metabolism, molecular chaperoning, calcium ion binding, proteolysis, immune regulation, protein transport, signal transduction, apoptosis, and cytoskeleton organization. The identified proteins were classified into seven major groups by using cluster analysis (analyzed with Cluster 3.0 software, and viewed with Treeview software) (Fig. 4).

\section{Immunoblotting validation for differentially expressed proteins}

In order to validate the data obtained from 2-DE, Western blot analysis of protein abundance changes in A549 cells was performed by immunoblotting. As shown in Fig. 5, EIF5A, PSMB6, ATP5A1 and ALDH1 were downregulated in periplocin treated cells, which was consistent with the 2-DE results.

\section{Analysis of the signaling network using bioinformatics}

We uploaded the 39 identified proteins along with their names into STRING. A merge network is shown in Fig. 6. Of the 39 identified proteins, 34 were interconnected and 5 proteins did not show any link at the chosen confidence level (STRING score $=0.4$ ). The most significant functions associated with this network were transcription, metabolism, proteolysis, electron transport, and molecular chaperoning. EIF3I, EIF5A, USP5 and RPLP2 are important proteins related to transcription; PSMA3, PSMB6 and PSMB4 are important proteins involved inproteasome-related proteolysis. These proteins are related to two major signal nets, transcription and proteolysis, as shown in Fig. 6 . The network view summarizes the network of predicted associations for a particular group of proteins. 
Fig. 3. Identification of protein spot \#7. (A) Peptide mass fingerprinting (PMF) of protein ATP5A1. (B) MS/ MS spectrum of peak $[\mathrm{M}+2 \mathrm{H}]^{2+}$ at $\mathrm{m} / \mathrm{z} 812.9689$ matched sequence R.TGAIVDVPVGEELLGR.F of ATP5A1.

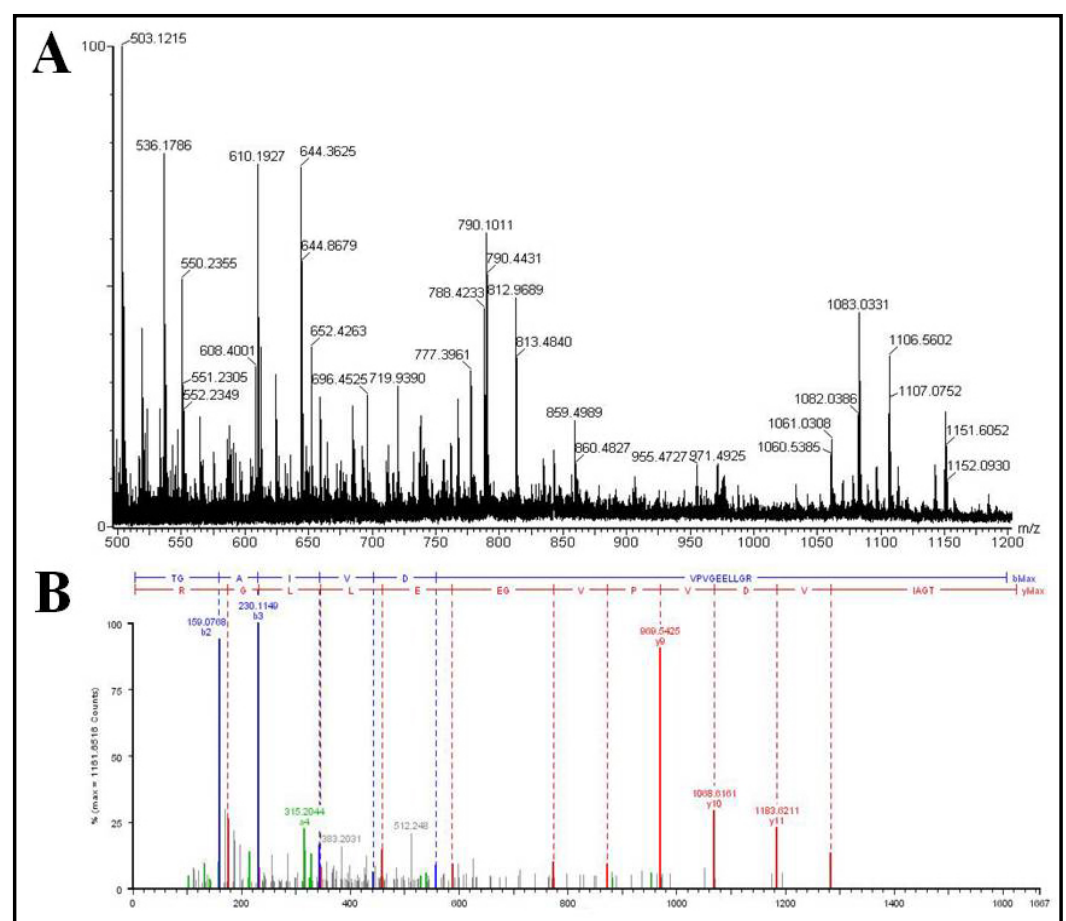

Fig. 4. Functional clustering analysis of the identified proteins from A549 cells treated with periplocin and NS. The fold changes of these proteins were assigned into diverse functional categories mainly based on Gene Ontology biological processes. Expression of proteins in the normal group was constant at 0 , proteins upregulated were in red, and the down-regulated proteins were in green. The intensity of the color green or red corresponds to the degree of alteration, respectively, according to the color strip at the right of the figure. A pie chart represented that 39 dysregulated proteins were classified into 7 groups with diverse functions including metabolism (38.5\%), molecular chaperoning (12.8\%), protein transport $(15.4 \%)$ and other functions (33.3\%).



\section{Discussion}

Accumulating preclinical and clinical data suggest that the cardiac glycosides have excellent activity against a variety of solid tumors $[11,12]$. Periplocin, one of the cardiac 


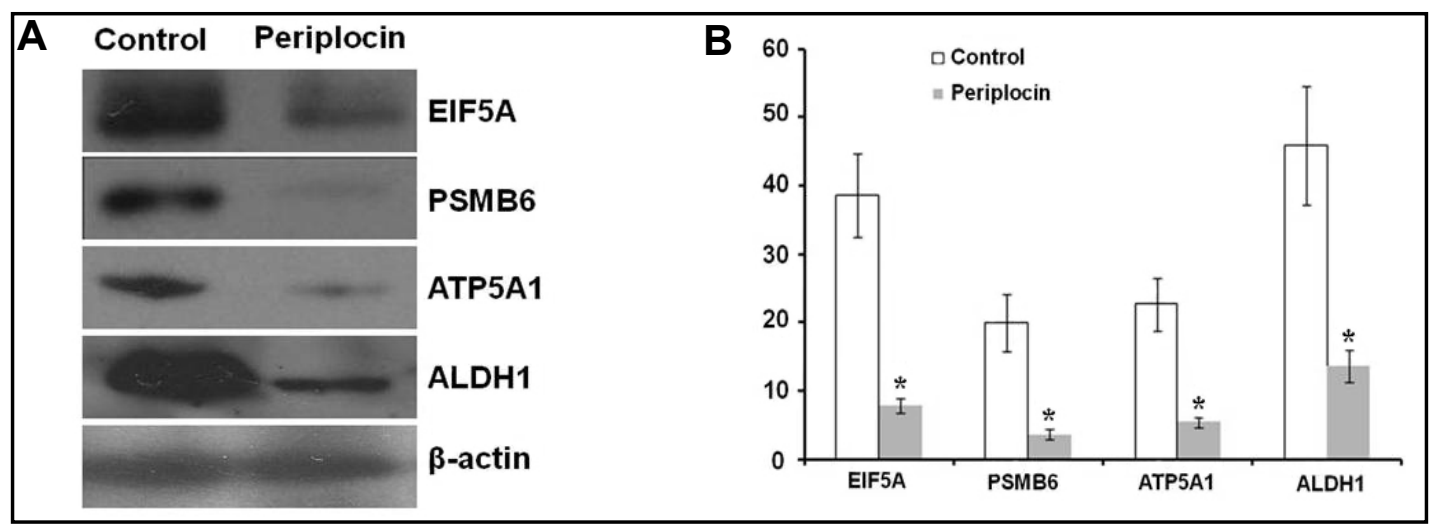

Fig. 5. Western blot for the expression of EIF5A, PSMB6, ATP5A1 and ALDH1. (A) EIF5A, PSMB6, ATP5A1 and ALDH1 were down-regulated in periplocin treated A549 cells. (B) Western blot data were quantified densitometrically and $\beta$-actin in the experiment was used as a control. Data are expressed as mean \pm SD from three independent experiments. ${ }^{*} \mathrm{p}<0.05$, compared with control group.

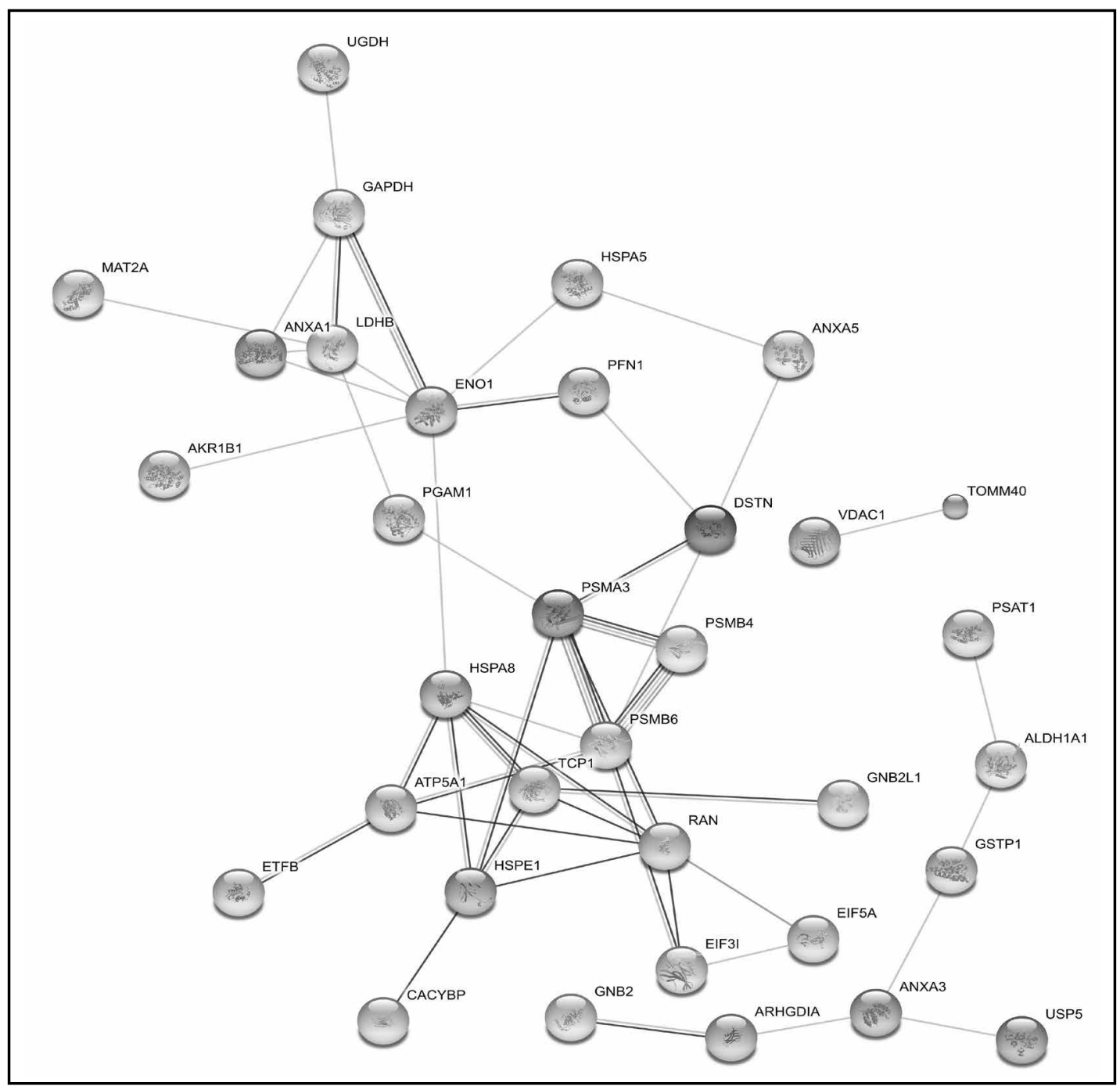

Fig. 6. Prediction of signaling networks between dysregulated proteins in periplocin-treated A549 cells. The identified differentially expressed proteins were analyzed with the STRING tool. In this network, nodes are proteins. Hovering over a node will display its annotation; the lines represent the predicted functional associations. The number of lines represents strength of predicted functional interactions between proteins. 
glycosides, was derived from cortex periplocae mainly used for treatment of rheumatoid arthritis, reinforcement of bones and tendons and alleviation of ache in lumbus and knee. It was reported that periplocin could strongly induce p16 promoter activity at low concentration, and showed high permeability and can be absorbed in rat intestine [13, 14]. Some researchers have shown that periplocin has strong inhibitory effects on the proliferation of many tumor cell lines in vitro, such as SW480, SMC-7721, TE13, BGC-823, MCF-7, PC3 and so on $[15,16]$. Our previous data suggested that periplocin exhibited antitumor activity both in human and mouse lung cancer xenograft models by inhibiting AKT/ERK signaling pathways, downregulating cyclin D1 and Bcl-2, and increasing expression of Bax [7]. However, the molecular targets through which periplocin acts have not yet been fully determined because of the complex anti-cancer mechanisms underlying the natural products.

In the present study, lung cancer cell lines A549 were used as a model, and a 2DEbased proteomic approach was undertaken to annotate the protein species whose levels are altered in A549 cells after treatment with periplocin. The proteomic analysis detected a total of 39 altered protein species, whose functions are connected with diverse biological processes such as metabolism, transcription, signal transduction, proteolysis and molecular chaperoning. Among them, 29 protein species were down-regulated and 10 protein species were up-regulated. Periplocin mediated down-regulation of the oncoproteins, ATP5A1, EIF5A, ALDH1 and so on, are thought to be associated with the antitumor activity of periplocin in A549 cells.

F1Fo-ATP synthase comprises a soluble F1 portion and a membrane-spanning Fo portion. For every three to four protons that are released into the matrix of mitochondria from the intermembrane space, one ATP is synthesized. However, ATP synthase components have recently been identified as cell-surface receptors for apparently unrelated ligands in the course of studies carried out on angiogenesis, lipoprotein metabolism, innate immunity, hypertension or regulation of food intake [17-20]. Ectopic F1Fo-ATP synthase was thought to be a potential marker for tumor target therapy [21-23]. Our findings have also shown that ATPB on the cell surface may be a potential biomarker and therapeutic targets for the immunotherapy of non-small cell lung cancer (NSCLC) [21-23]. Besides, over-expression of ATP synthase a-subunit may be involved in the progression and metastasis of breast cancer, representing a potential biomarker for diagnosis, prognosis and a therapeutic target for breast cancer [24]. Down-regulation of ATP synthase ecto- $\alpha$-subunit (ATP5A1) may play a role in periplocin-mediated growth inhibition of A549 cells, as well as induction of antiangiogenesis.

Another protein species found to be altered by periplocin treatment is EIF5A. EIFs play an important role in eukaryotic translation. EIF5A is thought to function as a nucleocytoplasmatic shuttle protein. It has been well established to be essential for sustained cell proliferation in mammalian cells; while inhibiting of EIF5A activation would exert strong antiproliferative effects in various human cancer cell lines, and cause arrest of cell cycle progression $[25,26]$. EIF5A plays an important role in hepatocellular carcinoma tumorigenesis and metastasis, and targeting eIF5A hypusination by inhibitor or eIF5A2 by RNA interference (RNAi) may offer new therapeutic alternatives to hepatocellular carcinoma patients [27]. Moreover, overexpression of eIF5A-2 might play an important role in carcinogenesis of NSCLC, and might serve as a useful molecular marker for those with stage I disease [28]. In the present study, we found eIF5A to be down-regulated with periplocin treatment.

ALDH1 was down-regulated after periplocin treatment. ALDH1 is a cytosolic enzyme which catalyzes the irreversible oxidation of retinol to retinoic acid and is thought to play a role in cellular differentiation via the retinoid pathway [29]. ALDH1 has been shown to regulate hematopoiesis by promoting myeloid differentiation [30]. Besides, ALDH1 is a marker of normal and malignant human mammary stem cells and a predictor of poor clinical outcome [31]. In addition, recent researches have demonstrated ALDH1 activity is a functional marker for lung cancer [32, 33]. 
In the present study, using proteomic tools 39 protein species were found to be altered in lung cancer cell lines A549 following periplocin treatment. Further studies will be needed to answer the important questions, namely mechanisms that expressions of ATPB and EIF5A are regulated by periplocin. At the same time, additional studies need to be conducted to elucidate the possible crosstalk among the affected protein species and their potential relationship to the inproteasome-related proteolysis processes, which have been shown to play important roles in the pleiotropic effects of periplocin.

\section{References}

1 Yu Y, Xu X, Du Z, Shi M: Non-platinum regimens of gemcitabine plus docetaxel versus platinum-based regimens in first-line treatment of advanced non-small cell lung cancer: a meta-analysis on 9 randomized controlled trials. Cancer Chemother Pharmacol 2012;69:1265-1275.

- Wang S, Wu X, Tan M, Gong J, Tan W, Bian B, Chen M, Wang Y: Fighting fire with fire: poisonous Chinese herbal medicine for cancer therapy. J Ethnopharmacol 2012;140:33-45.

3 Newman RA, Yang P, Pawlus AD, Block KI: Cardiac glycosides as novel cancer therapeutic agents. Mol Interv 2008;8:36-49.

4 Mijatovic T, Van Quaquebeke E, Delest B, Debeir O, Darro F, Kiss R: Cardiotonic steroids on the road to anticancer therapy. Biochim Biophys Acta 2007;1776:32-57.

-5 Winnicka K, Bielawski K, Bielawska A, Surazyński A: Antiproliferative activity of derivatives of ouabain, digoxin and proscillaridin A in human MCF-7 and MDA-MB-231 breast cancer cells. Biol Pharm Bull 2008;31:1131-1140.

6 Mekhail T, Kaur H, Ganapathi R, Budd GT, Elson P, Bukowski RM: Phase 1 trial of Anvirzel in patients with refractory solid tumors. Invest New Drugs 2006;24:423-427.

-7 Lu ZJ, Zhou Y, Song Q, Qin Z, Zhang H, Zhou YJ, Gou LT, Yang JL, Luo F: Periplocin inhibits growth of lung cancer in vitro and in vivo by blocking AKT/ERK signaling pathways. Cell Physiol Biochem 2010;26:609618.

8 Lu ZJ, Liu SY, Yao YQ Zhou YJ, Zhang S, Dai L, Tian HW, Zhou Y, Deng HX, Yang JL, Luo F: The effect of miR-7 on behavior and global protein expression in glioma cell lines. Electrophoresis 2011;32:3612-3620.

\ Lu ZJ, Song QF, Jiang SS, Song Q Wang W, Zhang GH, Kan B, Chen LJ, Yang JL, Luo F, Qian ZY, Wei YQ Gou LT: Identification of ATP synthase beta subunit (ATPB) on the cell surface as a non-small cell lung cancer (NSCLC) associated antigen.BMC Cancer 2009;9:16.

10 von Mering C, Jensen LJ, Kuhn M, Chaffron S, Doerks T, Krüger B, Snel B, Bork P: STRING 7--recent developments in the integration and prediction of protein interactions. Nucleic Acids Res 2007;35:D358362 .

11 Schoner W, Scheiner-Bobis G: Endogenous and exogenous cardiac glycosides: their roles in hypertension, salt metabolism, and cell growth. Am J Physiol Cell Physiol 2007;293:C509-536.

12 Bagrov AY, Shapiro JI, Fedorova OV: Endogenous cardiotonic steroids: physiology, pharmacology, and novel therapeutic targets. Pharmacol Rev 2009;61:9-38.

13 Egawa K, Kurihara Y, Ito T, Matsumoto M, Nose K: Induction of p16INK4a transcription and of cellular senescence by aclacinomycin-derivatives and cardiac glycosides. Biol Pharm Bull 2002;25:461-465.

14 Xie YS, Ren XL, Pan GX, Gao XM, Liu CX: The assessment of absorption of periplocin in situ via intestinal perfusion of rats by HPLC. Biomed Chromatogr 2008;22:196-201.

15 Du YY, Liu X, Shan BE: Periplocin extracted from cortex periplocae induces apoptosis of SW480 cells through inhibiting the Wnt/beta-catenin signaling pathway. Ai Zheng 2009;28:456-460.

16 Zhao L, Shan B, Du Y, Wang M, Liu L, Ren FZ: Periplocin from Cortex periplocae inhibits cell growth and down-regulates survivin and c-myc expression in colon cancer in vitro and in vivo via beta-catenin/TCF signaling. Oncol Rep 2010;24:375-383.

17 Champagne E, Martinez LO, Collet X, Barbaras R: Ecto-F1Fo ATP synthase/F1 ATPase: metabolic and immunological functions. Curr Opin Lipidol 2006;17:279-284.

18 Chi SL, Wahl ML, Mowery YM, Shan S, Mukhopadhyay S, Hilderbrand SC, Kenan DJ, Lipes BD, Johnson CE, Marusich MF, Capaldi RA, Dewhirst MW, Pizzo SV: Angiostatin-like activity of a monoclonal antibody to the catalytic subunit of F1F0 ATP synthase. Cancer Res 2007;67:4716-4724. 
19 Malaval C, Laffargue M, Barbaras R, Rolland C, Peres C, Champagne E, Perret B, Tercé F, Collet X, Martinez LO: RhoA/ROCK I signalling downstream of the P2Y13 ADP-receptor controls HDL endocytosis in human hepatocytes. Cell Signal 2009;21:120-127.

20 Vantourout P, Martinez LO, Fabre A, Collet X, Champagne E: Ecto-F1-ATPase and MHC-class I close association on cell membranes. Mol Immunol 2008;45:485-492.

21 Ruan Y, Wan M: An optimized procedure for solubilization, reduction, and transfer of human breast cancer membrane-enriched fraction by 2-DE. Electrophoresis 2007;28:3333-3340.

-22 Huang TC, Chang HY, Hsu CH, Kuo WH, Chang KJ, Juan HF: Targeting therapy for breast carcinoma by ATP synthase inhibitor aurovertin B. J Proteome Res 2008;7:1433-1444.

-23 Ma Z, Cao M, Liu Y, He Y, Wang Y, Yang C, Wang W, Du Y, Zhou M, Gao F: Mitochondrial F1Fo-ATP synthase translocates to cell surface in hepatocytes and has high activity in tumor-like acidic and hypoxic environment. Acta Biochim Biophys Sin 2010;42:530-537.

24 Pan J, Sun LC, Tao YF, Zhou Z, Du XL, Peng L, Feng X, Wang J, Li YP, Liu L, Wu SY, Zhang YL, Hu SY, Zhao WL, Zhu XM, Lou GL, Ni J: ATP synthase ecto- $\alpha$-subunit: a novel therapeutic target for breast cancer. J Transl Med 2011;9:211.

25 Li AL, Li HY, Jin BF, Ye QN, Zhou T, Yu XD, Pan X, Man JH, He K, Yu M, Hu MR, Wang J, Yang SC, Shen BF, Zhang $\mathrm{XM}$ : A novel eIF5A complex functions as a regulator of p53 and p53-dependent apoptosis. J Biol Chem 2004;279:49251-49258.

26 Kaiser A: Translational control of eIF5A in various diseases. Amino Acids 2012;42:679-684.

-27 Lee NP, Tsang FH, Shek FH, Mao M, Dai H, Zhang C, Dong S, Guan XY, Poon RT, Luk JM: Prognostic significance and therapeutic potential of eukaryotic translation initiation factor 5A (eIF5A) in hepatocellular carcinoma. Int J Cancer 2010;127:968-976.

-28 He LR, Zhao HY, Li BK, Liu YH, Liu MZ, Guan XY, Bian XW, Zeng YX, Xie D: Overexpression of eIF5A-2 is an adverse prognostic marker of survival in stage I non-small cell lung cancer patients. Int J Cancer 2011;129:143-150.

-29 Resetkova E, Reis-Filho JS, Jain RK, Mehta R, Thorat MA, Nakshatri H, Badve S: Prognostic impact of ALDH1 in breast cancer: a story of stem cells and tumor microenvironment. Breast Cancer Res Treat 2010;123:97108.

-30 Rice KL, Izon DJ, Ford J, Boodhoo A, Kees UR, Greene WK: Overexpression of stem cell associated ALDH1A1, a target of the leukemogenic transcription factor TLX1/HOX11, inhibits lymphopoiesis and promotes myelopoiesis in murine hematopoietic progenitors. Leuk Res 2008;32:873-883.

-31 Ginestier C, Hur MH, Charafe-Jauffret E, Monville F, Dutcher J, Brown M, Jacquemier J, Viens P, Kleer CG, Liu S, Schott A, Hayes D, Birnbaum D, Wicha MS, Dontu G: ALDH1 is a marker of normal and malignant human mammary stem cells and a predictor of poor clinical outcome. Cell Stem Cell 2007;1:555-567.

- 32 Ucar D, Cogle CR, Zucali JR, Ostmark B, Scott EW, Zori R, Gray BA, Moreb JS: Aldehyde dehydrogenase activity as a functional marker for lung cancer. Chem Biol Interact 2009;178:48-55.

33 Liang D, Shi Y: Aldehyde dehydrogenase-1 is a specific marker for stem cells in human lung adenocarcinoma. Med Oncol 2012;29:633-639. 